\title{
The role of categorical information in processing relational attributes
}

\author{
KEVIN M. SAILOR \\ Lehman College, City University of New York, Bronx, New York \\ and \\ EDWARD J. SHOBEN \\ University of Illinois at Urbana-Champaign, Urbana, Illinois
}

\begin{abstract}
In two experiments, the effect of knowledge about relevant common taxonomic categories on judgments of relative magnitude was investigated. In Experiment 1, subjects judged the relative size of objects that belonged to the categories building and animal. The symbolic distance effect was smaller for between-category pairs (e.g., horse-cabin) whose members could be classified more quickly into the two categories. This result strongly suggests that subjects used category membership to determine relative size on at least some portion of the trials. In Experiment 2, a similar attenuation of symbolic distance was obtained when subjects judged the relative height of furniture and vehicles. A simple explanation of the reduced symbolic distance effect for between-category pairs is that subjects occasionally fail to categorize the items into different categories and have to compute relative magnitude from the magnitudes of the two items. Fits to the data using the bootstrap technique suggest that more involved assumptions are required.
\end{abstract}

A familiar issue confronting cognitive psychologists is the problem that the same information can be represented in a variety of states that reflect a more or less explicit representation of some fact about the world (Smith, 1978). For example, some models of semantic memory assume that category membership is computed from knowledge about the attributes of their exemplars, but other models assume that category membership is explicitly represented and retrieved directly (Chang, 1986; Smith, 1978).

Within the area of comparative judgment, the predominant view has been that relative magnitude is computed (Banks, 1977; Holyoak, 1978; Moyer, 1973). The main reason that researchers have believed that relative magnitude is computed is the robust phenomenon of the symbolic distance effect. The symbolic distance effect is the finding that the time required to select the lesser or greater of two objects increases as they become more similar on a specified dimension. For example, it is easier to determine that a car is larger than a shoe than it is to determine that a car is larger than a piano. This finding suggests that relative magnitude is determined by evaluating differences in the absolute magnitudes of objects because there is no reason to believe that response time (RT) would be a function of symbolic distance if the order of a pair was explicitly represented.

Subsequently, the notion that relative magnitude is always computed has been challenged with the demonstra-

This research was supported, in part, by Grant 2S06GM08225 from $\mathrm{NIH}$ to Lehman College and by Grant BN586-08215 from the National Science Foundation to the second author. Correspondence should be addressed to K. M. Sailor, Department of Psychology, Lehman College, CUNY, 250 Bedford Park Blvd. West, Bronx, NY 10468-1589 (e-mail: kmslc@cunyvm.cuny.edu). tion of categorization effects (Brown \& Siegler, 1991; Kosslyn, Murphy, Bemesderfer, \& Feinstein, 1977; Maki, 1981; Pliske \& Smith, 1979; Sailor \& Shoben, 1993). Categorization effects refer to the finding that the symbolic distance effect is either reduced or eliminated if a relevant categorical distinction is correlated with relative magnitude. For example, Pliske and Smith (1979) had subjects learn a set of names (half male and half female) that was ordered on intelligence. In the critical experimental group, gender and intelligence were correlated because all of the names of one gender were brighter than all of the names of the other gender. Subjects then judged which of two names was more intelligent. When subjects judged pairs with members of the same gender (i.e., within-category pairs), pairs of names that were closer in intelligence were more difficult to discriminate than were pairs that were farther apart in the order. In contrast, Pliske and Smith found that the distance effect was greatly reduced when subjects judged pairs that included a male and a female name (i.e., between-category pairs). This interaction suggests that people were categorizing the names as male or female and responding on the basis of that categorization.

The importance of these categorization effects is that they suggest that subjects can employ two distinct strategies when determining relative magnitude: a magnitude comparison process and a categorization process. The reason that these results suggest two distinct strategies is that it is difficult to reconcile performance on within-category pairs and between-category pairs if subjects did not use distinct strategies. For example, if subjects retrieved the modal size of the members of a category and used this value in lieu of the absolute magnitude of the individual exemplars as an input to the normal comparison process, 
then the same value would be retrieved for every member of a common taxonomic category. This coding would make it very difficult to discriminate between withincategory pairs because they would be identically coded, and, at the same time, it would eliminate the distance effect for between-category pairs because any comparison of the members of two different categories would be computed from the same difference in absolute magnitudes. Thus, the fact that the symbolic distance effect is frequently attenuated but not eliminated for between-category pairs and that subjects are still quite good at solving withincategory pairs indicates that they are capable of using either category membership or knowledge about the absolute magnitude of individual items on any given trial.

The primary goal of previous research has been to develop an account of the magnitude comparison process (Banks, 1977; Holyoak \& Mah, 1982; Shoben, Cech, Schwanenflugel, \& Sailor, 1989). In contrast, we are interested in trying to identify how subjects use category membership as an alternative to computing relative magnitude and describing how this alternative strategy is incorporated with the strategy of computing relative magnitude.

\section{Processing Accounts}

There are at least two broad classes of models that postulate two distinct states or processes leading to a response: sequential models and parallel models (Kosslyn et al., 1977).

These models differ in the sequencing of each process. Sequential models propose that the categorization process is invoked first. It runs to completion, before the magnitude comparison operation is begun (if it is needed). According to this account, we obtain a robust distance effect for within-category pairs because the categorization strategy does not yield an answer. In its simplest form, the sequential model predicts that the symbolic distance effect should be eliminated for between-category pairs because the categorization process always produces the response (Kosslyn et al., 1977). In contrast, the version of the sequential model that we consider assumes that the distance effect is attenuated for between-category pairs, rather than eliminated, because the categorization process does not always produce an answer. We will refer to this model as the mixture model. ${ }^{1}$

In contrast to the mixture model, parallel models assume that the categorization and comparison processes run in parallel, and a response is made when one of the two processes terminates successfully. Distance effects are attenuated for between-category pairs because, on some occasions, the categorization process will finish before the magnitude comparison process.

\section{EXPERIMENT 1}

Although both of these two-state models can account for categorization effects, the strategy of contrasting the size of the distance effect for within-category and betweencategory pairs is a fairly weak test of these models, be- cause the test requires only that there is a very general qualitative distinction between within-category pairs and between-category pairs. The goal of Experiment 1 was to conduct a more stringent test of the hypothesis that the accessibility of a relevant categorical distinction should influence the size of the symbolic distance effect.

In this experiment, we devised a test of these two models on the basis of predictions that they make about the size of the distance effect for between-category pairs under different conditions. Both models predict that the betweencategory distance should be larger when items are difficult to categorize and smaller when they are easier to categorize into their respective categories. According to the mixture model, mean RT is the weighted average of the mean duration of categorizing and the mean duration of both categorizing and comparing the two objects. If the relative magnitude of a pair can be easily determined solely by the categorization process, then the mean of between-category pairs will approximate the mean duration of the categorization process. However, if it is difficult to categorize a pair, then the probability of determining their relative magnitude solely on the basis of the categorization process should decrease. Under these circumstances, it is likely that subjects will have to compare the items after they have attempted to categorize them, and one would expect to obtain distance effects that were quite large.

It is important to emphasize that the mixture model does not predict an attenuation of the distance effect, if one assumes that manipulating categorization difficulty influences the duration of the categorization process but not the probability of using that process. If an increase in the difficulty of categorizing items prolongs categorization but does not change the probability of using this process, then the effects of categorization difficulty and symbolic distance will be additive.

In the parallel race model, subjects attempt to categorize and compare the items simultaneously. The predictions of this model are determined by the typical duration of each response. If the categorization process is generally faster than the comparison process, then the categorization process is more likely to produce a response. If, however, comparing the items is generally faster than categorizing them, then the comparison process is more likely to produce a response. The race model predicts that the between-category distance effect is larger when the categorization process is prolonged, because a larger proportion of trials will be determined by the comparison process. Thus, items that are difficult to categorize should produce a larger distance effect, because they require more time to categorize than do easily categorized items.

All of the stimuli belonged to one of two categories: buildings and animals. The category dominance (Loftus $\&$ Scheff, 1971) or ease with which the category membership of these stimuli could be established was systematically varied. If stimuli are categorized into semantic categories as part of the decision process, then one would expect to find an attenuation of the distance effect that depended on category dominance. 


\section{Method}

Design. Two eight-term orderings were constructed. For each ordering, the four smallest items were animals and the four largest were buildings. For one ordering, all items were of low category dominance; for the other ordering, all items were of high category dominance. Category dominance was determined by performance in a pilot experiment in which a separate group of subjects was timed as they classified a group of animals and buildings. For the highcategory-dominance ordering, items were classified, on average, in $642 \mathrm{msec}$. Low-category-dominance items took $704 \mathrm{msec}$ to classify, on average. Magnitudes of the items were determined in a second pilot experiment in which subjects rated the magnitudes of 81 animals and buildings. These materials were presented in a single randomized list, and subjects rated their size on a 20-point scale.

Materials. The materials are presented in Table 1 . We tried to equate the low-dominance and high-dominance lists on a number of variables. The range in magnitude was 14.4 for the high-dominance list and 14.1 for the low-dominance list. Average word frequencies were 13.4 and 24.6, respectively, according to the Kučera and Francis (1967) norms. Mean word length was 6.8 letters for the highdominance list and 6.1 letters for the low-dominance list. Thus, if anything, the high-dominance items were slightly longer and slightly less frequent than were their low-dominance counterparts.

Procedure. In the first phase of the experiment, the subjects saw only within-category pairs. Items from both the high-dominance and the low-dominance lists were presented randomly, subject to the constraint that pairs were separated by a maximum of three ordinal steps. Each block consisted of 24 within-category pairs, which were presented once with each instruction. There were three such blocks.

In the second phase of the experiment, there were both betweencategory trials and within-category trials. For both the high-dominance and the low-dominance lists, all possible within-category pairs (separated by no more than three ordinal steps) were included. There were 12 such pairs from each list. All possible between-category pairs were used, subject to the constraint that no pair had members that were separated by more than four items (e.g., owl-store or rabbitrestaurant). For each list, there were 13 such pairs. The subjects were presented with two blocks of these 50 pairs.

Within each block, each pair was presented once with the instruction to select the smaller item and once with the instruction to select the larger item. Across the two blocks, each member appeared equally often on the left and right, counterbalanced with instruction. The subjects were given two 30 -sec breaks during the experiment.

The subjects were instructed that they would see pairs of items, preceded by a comparative (smaller? or larger?). They were to decide the correct answer and respond by pressing the " 5 " key on the terminal if the correct answer appeared on the left side of the screen and the " 9 " key if it appeared on the right side of the screen. The subjects were instructed to respond as quickly and as accurately as

Table 1

Size Ratings of Stimuli for Experiment 1

\begin{tabular}{|c|c|c|c|}
\hline \multicolumn{4}{|c|}{ Category Dominance } \\
\hline \multicolumn{2}{|c|}{ High } & \multicolumn{2}{|c|}{ Low } \\
\hline Stimulus & Rating & Stimulus & Rating \\
\hline \multicolumn{4}{|c|}{ Animal } \\
\hline Rabbit & 2.4 & Owl & 2.7 \\
\hline Turkey & 4.7 & Fox & 4.6 \\
\hline Octopus & 6.2 & Kangaroo & 6.4 \\
\hline Pony & 8.0 & Alligator & 8.2 \\
\hline \multicolumn{4}{|c|}{ Building } \\
\hline Woodshed & 10.2 & Cottage & 10.4 \\
\hline Restaurant & 12.2 & Store & 12.0 \\
\hline Theater & 14.6 & Library & 14.9 \\
\hline Castle & 16.8 & Stadium & 16.8 \\
\hline
\end{tabular}

possible. Prior to the start of the experiment, the subjects were told that the items would be examples from the categories animals and buildings.

Subjects. Twenty-eight undergraduates at the University of Illinois participated in partial fulfillment of a course requirement. All 28 subjects met a criterion of fewer than 35 errors ( $10 \%)$.

\section{Results and Discussion}

RTs greater than $3 S D$ s from the mean were excluded from the analysis and treated as errors. Errors $(.049)$ correlated positively with the RTs $(r=.67)$.

The results of primary interest are those from the second phase of the experiment in which both between-category and within-category comparisons were included. For within-category comparisons, only three levels of distance were present. Consequently, for within-category pairs, we used the RTs for adjacent comparisons (Split 1) to estimate small symbolic distance, and we used the RTs for pairs whose members were separated by two intervening items (Split 3) to estimate large symbolic distance. For between-category pairs, we collapsed two splits to obtain more stable data. We used Split 1 and Split 2 pairs to estimate small symbolic distance and Split 4 and Split 5 pairs to estimate large symbolic distance.

The critical prediction concerns the effect of category dominance on the diminution of the distance effect in between-category pairs. Consistent with previous findings (Kosslyn et al., 1977; Sailor \& Shoben, 1993), the distance effect in the within-category pairs was very large (355 and $444 \mathrm{msec}$ in the high-dominance and low-dominance orders, respectively). Also consistent with these findings, the distance effect was greatly attenuated for the highdominance between-category pairs. As Table 2 indicates, the distance effect was only $140 \mathrm{msec}$ for these pairs. Importantly, there was no such attenuation for low-dominance between-category pairs. Here, the distance effect was a robust $383 \mathrm{msec}$.

Surprisingly, given the magnitude of the effect, this three-way interaction among type of category pair, distance, and dominance was not significant $[F(1,27]=$ $\left.2.31, M S_{\mathrm{e}}=35,873, p=.14\right]$. An inspection of the item means suggests that this failure to reach significance can be attributed to the exceptionally long RT for one of the within-category low-split low-dominance items (kangaroo-alligator). This RT was $379 \mathrm{msec}$ longer than the mean for this condition and $259 \mathrm{msec}$ longer than the next longest RT in this condition. Moreover, more errors (.29) were made to this pair than to any other pair in Phase 1. When the RT for this item is removed, the distance effect for low-dominance within-category pairs is virtually identical $(370 \mathrm{msec})$ to the size of the distance effect for the same pairs in the high-dominance condition $(355 \mathrm{msec})$. Consequently, the data were reanalyzed with the kangaroo-alligator pair excluded. The three-way interaction among type of category pair, distance, and dominance was significant with kangaroo-alligator removed $\left[F(1,27)=4.69, M S_{\mathrm{e}}=38,458, p<.05\right]$, indicating that the degree to which the distance effect was attenuated was affected by category dominance. 
culation of the Split 1 within-category mean. The reason that times for these pairs were excluded was to equate the within-category and between-category Split 1 pairs for serial position.

In contrast to the two pairs with end terms in the withincategory condition, the Split 1 between-category pair was constructed from the two middle items in the order. This discrepancy is important because a common finding is that extreme pairs are responded to more quickly than are moderate pairs (Banks, 1977), and this advantage for extreme pairs is most pronounced for low-symbolicdistance pairs (Holyoak \& Patterson, 1981; Shoben et al., 1989). A comparison of the two end pairs $(\mu=1,123 \mathrm{msec})$ to the four middle within-category pairs ( $\mu=1,331 \mathrm{msec}$ ) indicates the presence of a serial position effect in the present data $[F(1,41)=85.4, p<.001]$. Therefore, it seemed reasonable to assume that these two pairs underestimated the comparison difficulty of the betweencategory pair, and we dropped them from the calculation of the Split 1 within-category mean.

An inspection of Table 4 indicates that the results are quite similar to previous findings: There was an overall distance effect that was much smaller for betweencategory pairs than it was for within-category pairs. Collapsed over between-category and within-category pairs, RT decreased with increasing symbolic distance. The differences between Split 1, Split 2, and Split 3 pairs were significant $\left[F(2,82)=106.2, M S_{\mathrm{e}}=8,907, p<.001\right]$. Although these differences were significant for betweencategory pairs $\left[F(2,82)=20.1, M S_{\mathrm{e}}=6,579, p<.001\right]$, the decrease in RT across the three levels of symbolic distance was larger for within-category pairs $(301 \mathrm{msec})$ than it was for between-category pairs $(113 \mathrm{msec})[F(2,82)=$ $\left.31.7, M S_{\mathrm{e}}=7,023, p<.001\right]$. These results indicate that the subjects were using category membership to decide the relative magnitude of between-category pairs on some portion of the trials.

The mixture model was tested by estimating the value of the mixing parameter, $p$. The terms of Equation 4 can be rearranged to express $p$ in terms of the ratio of the change in between-category times over the within-category times for two levels of symbolic distance:

$$
p=1-\left(\mu_{\mathrm{b} 1}-\mu_{b 2}\right) /\left(L_{1}-L_{2}\right) .
$$

In other words, the probability of categorizing the pair initially is 1 minus the between-category distance effect divided by the within-category distance effect. Three values of $p$ can be estimated from the data by comparing

Table 4

RTs (in Milliseconds) and Error Rates as a

Function of Category Membership and Distance

\begin{tabular}{lccccc} 
& \multicolumn{4}{c}{ Category Membership } \\
\cline { 2 - 3 } \multicolumn{1}{c}{ Distance } & RT & Error Rate & & RT & Error Rate \\
\cline { 2 - 3 } \cline { 5 - 6 } \multicolumn{1}{c}{ Split 1 } & 1,331 & .11 & & 1,189 & .03 \\
Split 2 & 1,130 & .03 & & 1,137 & .02 \\
Split 3 & 1,021 & .01 & & 1,076 & .02 \\
Distance effect & 224 & & 94 & \\
\hline
\end{tabular}

Splits 1 and 2, Splits 1 and 3, and Splits 2 and 3. These three estimates will be denoted $p_{12}, p_{13}$, and $p_{23}$, respectively. If the mixture model is a good account of the data, then all three of these estimates should agree and should fall between zero and one. On the other hand, substantial discrepancies in these three estimates would indicate that the model is inappropriate.

Although three estimates of $p$ can be calculated from the data, a violation of the mixture model will be most apparent in a comparison of the $p_{12}$ and $p_{23}$ estimates. According to Equation 4 , the three estimates of $p$ will agree only if the between-category distance effect is a constant proportion of the within-category distance effect across all three levels of symbolic distance. If the proportions used to calculate the $p_{12}$ and $p_{23}$ estimates differ, then the estimate of $p_{13}$ must be of intermediate value, because the Split 1-Split 3 distance effect is the sum of the Split 1-2 and Split 2-3 distance effects. This means that if $p_{12}$ is greater than the $p_{23}$ estimate, then the $p_{13}$ estimate will also be larger than the $p_{23}$ estimate. Thus, a comparison of the $p_{12}$ and $p_{23}$ estimates provides a better idea of whether distance effects in the between-category condition are a constant proportion of the within-category distance effects than does a comparison of one of these estimates to an estimate to which both distance effects contribute.

Instead of fitting the mixture model to individual data, the model was fit to group data using a technique known as the bootstrap (Diaconis \& Efron, 1983). The reason that the model was fit to group data is that small amounts of error can affect estimates of $p$ from individual data when the true value of the distance effect is small. For example, a comparison of Split 2 and Split 3 means suggests that the true value of the between-category distance effect is $61 \mathrm{msec}$, and the within-category distance effect is $109 \mathrm{msec}$. However, given the fact that the standard deviation of the Split 2 versus Split 3 between-category distance effect is $99 \mathrm{msec}$, the observed distance effects of individual subjects varies substantially. Although some of this variation is the result of individual differences in processing speed that would also be reflected in the size of each subject's within-category distance effect, some of this variation must be attributed to error. If the observed between-category distance effect is $1 S D$ faster than the true distance effect, then $p$ would be 1.34; however, if the observed distance effect was $1 S D$ slower than the true distance effect, then the observed value of $p$ would be only -0.47 . Moreover, this instability is compounded if the variability in within-category distance effects is taken into account.

Assuming that mean RT provides a more reliable estimate of the mean duration of each process than does the mean RT of individual subjects, more reliable estimates of $p$ can be obtained by fitting the mixture model to group data. ${ }^{2}$ The bootstrap was used to determine the reliability of these estimates. The bootstrap is a computer-intensive technique that is used to create an empirically generated sampling distribution (Diaconis \& Efron, 1983; Noreen, 1989; Thompson, 1992; Wasserman \& Bockenholt, 1989). This empirical sampling distribution is created by ran- 
domly sampling with replacement from the original data set to create a large number of samples of the same size as the original data set. The statistic of interest is then calculated for each of these samples to create the empirical sampling distribution. This empirically generated sampling distribution is assumed to approximate the true sampling distribution of the statistic.

The value of this approach in the present circumstance is that it would not be possible to determine the reliability of our results without numerous repetitions of the experiment unless we were willing to assume a particular distribution for $p$. However, with the bootstrap, it is possible to determine whether the estimates of $p$ that were calculated for our particular set of subjects are likely to generalize to the population as a whole. The distributions of the $p_{12}$ and $p_{23}$ estimates of $p$ were calculated as follows. First, a set of 42 subjects was drawn randomly with replacement from the data set to create a sample of the same size as the original data set. Next, the RT for each of the six conditions was averaged over these subjects. Finally, one of the two different estimates of $p$ was calculated from these group averages. This process was repeated 1,000 times to generate 1,000 estimates of the $p_{12}$ estimate and repeated 1,000 times to generate 1,000 estimates of $p_{23}$.

The mean, standard deviation, and confidence intervals for each estimate of $p$ appear in Table 5. The confidence intervals were obtained by dropping the largest 25 and smallest 25 sample values for each of the three estimates of $p$ to leave an interval that contained $95 \%$, or 950 of the 1,000 sample values (Wasserman \& Bockenholt, 1989).

The null hypothesis was evaluated by comparing each of the $p_{12}$ estimates with every $p_{23}$ estimate and calculating the proportion of cases in which the $p_{12}$ estimate was greater than the $p_{23}$ estimate. This procedure is similar to the jackknife and cross-validation techniques (Wasserman \& Bockenholt, 1989). The advantage of this resampling plan is that the two estimates do not depend on the same Split 2 means because each estimate is calculated from separate samples rather than from the same sample. The importance of this independence is that one would expect to find that the $p_{12}$ estimate would be larger than the $p_{23}$ estimate in approximately $50 \%$ of the pairs if the two estimates were sampled from the same distribution. On the other hand, one would expect to find a consistent ordering of these two estimates over a large number of samples only if the two estimates were drawn from different populations. Thus, this resampling plan makes it possible to establish the probability of drawing a sample in which $p_{12}$ is greater than $p_{23}$ without making some of the

Table 5

Means, Standard Deviations, and Confidence Intervals for Estimates of the Mixing Parameter, $p$, for Experiment 2

\begin{tabular}{cccc}
\hline Estimate of $p$ & $M$ & $S D$ & $\begin{array}{c}\text { Confidence } \\
\text { Intervals }\end{array}$ \\
\hline$p_{12}$ & .74 & .098 & $.53<\mu<.92$ \\
$p_{23}$ & .44 & .137 & $.16<\mu<.72$ \\
\hline
\end{tabular}

parametric assumptions entailed in using the standard deviation of the bootstrapped samples to conduct a $t$ test.

The proportion of $p_{12}$ estimates that were greater than $p_{23}$ was .958 . This finding indicates that it is very unlikely that the two different estimates of $p$ were sampled from the same population. Although an exact value cannot be placed on this probability because the bootstrap is an approximation to the true sampling distribution, this proportion appears to be quite stable. We repeated the resampling plan an additional 99 times and found that the proportion ranged from .954 to .969 , with a mean of $.961 .^{3}$ These results indicate that the decrease in RT for betweencategory pairs is not a constant proportion of the RT of comparable within-category pairs and that the mixture model does not provide a good account of the data.

\section{Fits to Previous Experiments}

An important limitation of the preceding experiment is that the conclusions depend on the results obtained from a single set of items. As Clark (1973) noted, it is risky to assume that different conditions constitute unique levels of a fixed factor when a number of linguistic tokens are assigned to each condition. For example, the Split 1 between-category mean was calculated from RTs for one pair (e.g., car-dresser). Therefore, we fit the mixture model to data from three experiments that were presented in an earlier study (Sailor \& Shoben, 1993) to bolster the claims made for the results of Experiment 2.

In all three experiments, the subjects were presented with items selected from a 10-term linear order. Each item belonged to one of two nonoverlapping categories. Instead of determining which of two items was taller, the subjects determined which of two items was larger or smaller. In all other respects, the procedure in these experiments was quite similar to the procedure followed in Experiment 2. As was the case in Experiment 2, three levels of symbolic distance for within-category and betweencategory pairs could be compared. The means and error rates for these six conditions for each of the experiments appear in Table 6.

A detailed discussion of the results of these three experiments appears in the original study, but an inspection of Table 6 indicates that the results were quite similar to the results of the present Experiment 2. There was a significant attenuation of the distance effect for betweencategory pairs in all three experiments. More importantly, estimates of the mixing parameter calculated from the condition means presented in Table 6 are quite similar to the pattern observed in Experiment 2 of the present study. These estimates are presented in Table 7 . In all three experiments, the $p_{12}$ estimate was substantially larger than the $p_{23}$ estimate. ${ }^{4}$

The reliability of this difference was assessed using the resampling plan outlined above. The number of subjects in each of these experiments was substantially smaller than the number of subjects included in the present study. For this reason, the data from the three experiments were combined to create one data set with 70 subjects. This aggregation of the data from the three experiments seemed 
Table 6

RTs (in Milliseconds) and Error Rates as a Function of Category Membership and Distance in Sailor and Shoben (1993)

\begin{tabular}{|c|c|c|c|c|c|c|}
\hline \multirow[b]{3}{*}{ Distance } & \multicolumn{6}{|c|}{ Experiment From Sailor and Shoben (1993) } \\
\hline & \multicolumn{2}{|r|}{1} & \multicolumn{2}{|c|}{2} & \multicolumn{2}{|c|}{4} \\
\hline & RT & Error Rate & RT & Error Rate & $\mathrm{RT}$ & Error Rate \\
\hline \multicolumn{7}{|c|}{ Within-Category Pairs } \\
\hline Split 1 & 1,620 & .07 & 1,635 & .11 & 1,704 & .12 \\
\hline Split 2 & 1,445 & .03 & 1,456 & .05 & 1,464 & .04 \\
\hline Split 3 & 1,362 & .04 & 1,354 & .04 & 1,339 & .02 \\
\hline Distance effect & 258 & & 281 & & 365 & \\
\hline \multicolumn{7}{|c|}{ Between-Category Pairs } \\
\hline Split 1 & 1,550 & .04 & 1,437 & .08 & 1,548 & .04 \\
\hline Split 2 & 1,562 & .06 & 1,415 & .06 & 1,551 & .07 \\
\hline Split 3 & 1,528 & .06 & 1,381 & .02 & 1,472 & .03 \\
\hline Distance effect & 22 & & 56 & & 76 & \\
\hline
\end{tabular}

Note-The data presented for Experiment 1 are from the consistent condition.

justified because the materials were quite similar, and the results were quite similar. In fact, the same materials were used in Experiments 1 and 2, the materials in Experiment 4 were selected from the same norms, and the manipulation of symbolic distance was quite comparable to Experiments 1 and 2.

The proportion of $p_{12}$ estimates that was greater than the $p_{23}$ estimates was .944. This finding indicates that it is very unlikely that the two different estimates of $p$ were sampled from the same population. We repeated the resampling plan an additional 99 times and found that the proportion ranged from .932 to .952 with a mean of .940 . These results indicate that it is quite unlikely that the $p_{12}$ estimate would be smaller than the $p_{23}$ estimate in a replication of these experiments. Thus, the results of fits to these experiments indicate that the results of Experiment 2 cannot be attributed to the particular items that were employed. Instead, they suggest that RT for betweencategory pairs is not a constant proportion of RT for within-category pairs.

\section{GENERAL DISCUSSION}

The results of the two experiments replicate and extend previous findings in several respects. In previous research on the topic of categorization effects in comparative judgment, the claim that category membership could be used to determine relative magnitude was inferred from the finding that the distance effect was smaller from between-category pairs. Experiment 1 provides additional evidence for this claim by demonstrating that categorization effects are smaller for items that are categorized more slowly. Experiment 2 provides further evidence that these categorization effects are fairly robust by extending these findings to a new dimension and new categories.

This use of categorical information to decide relative magnitude may seem odd at first glance, but, in fact, it is very common. For example, we do not find it odd to answer the question, "Are dogs bigger than cats?" Even though we recognize the fact that there are some dogs that are actually smaller than most cats, this statement seems reasonable because we understand that we should not really worry about the differences among the individual exemplars of each category. In Experiment 2, we asked subjects about the heights of individual exemplars of the categories furniture and vehicles. Once they learned that all of the furniture in the experiment was shorter than all of the vehicles, then they knew it was safe to operate at the categorical level. Thus, when they saw a furniture-vehicle pair, they could simply respond on the basis that "furniture is shorter than vehicles."

There are several reasons why it may be advantageous for subjects to operate at the categorical level. In many cases, it is probably easier to operate at the categorical level because this information is more accessible. For example, it is almost certainly the case that subjects are more familiar with the general size of members of the category animal than they are with the sizes of many exemplars of this category. A second reason to operate at the categorical level is that the difference between the modal values of a category may be easier to work with than the frequently smaller differences between individual exemplars of the category. For example, the average size of an animal was 5.61 scalar units and the average size of a building was 11.68 scalar units in Experiment 1 of Sailor and Shoben (1993). This difference of approximately 6 scalar units was substantially larger than the average difference in the rated sizes of individual pairs of Splits $1-4$ ( $\mu=$ 3.06 scalar units). Therefore, it would have been easier to compare the modal sizes of the categories than to choose the larger of a given pair of exemplars from two categories, because the differences in the modal values of the category are frequently larger than the differences between individual exemplars. Clearly, the smaller size difference for individual exemplars would decline or disappear if the full range of exemplars in each category were compared, but the advantage of using category level information would probably remain. The rate at which RT decreases with increasing difference in the magnitudes of two items diminishes markedly (Holyoak \& Mah, 1982). This decrease in rate means that the advantage of using category level information for close pairs might outweigh any possible advantage for extremely remote pairs.

In this paper, we presented a test of a two-state mixture model. According to this model, subjects first attempt to determine relative magnitude by categorizing the items, but, if this process fails, they compute relative magnitude from knowledge about the absolute magnitude of the two items. This model assumes that either process is capable of determining relative magnitude for between-category pairs but that only the comparison process is capable of

Table 7

Observed Values of $p$ for Experiments 1, 2, and 4 in Sailor and Shoben (1993)

\begin{tabular}{cccc}
\hline & \multicolumn{3}{c}{ Experiment } \\
Estimate of $p$ & $1(n=26)$ & $2(n=18)$ & $4(n=26)$ \\
\hline$p_{12}$ & 1.07 & .88 & 1.01 \\
$p_{23}$ & .59 & .67 & .37
\end{tabular}


determining the relative magnitude of within-category pairs. The basic prediction of this model is that mean RT for between-category pairs should be a constant proportion of mean RT for within-category pairs. We tested this prediction by estimating the mixing parameter, $p$, at different levels of symbolic distance for the data from a number of previously published studies and from a new experiment. In every case, we found that the proportion of between-category time to within-category time was smaller for pairs whose members were closer in magnitude than for pairs whose magnitudes were more discrepant. This finding strongly suggests that the categorization and comparison processes are not strictly sequential.

The only potential caveat to this conclusion is that our test of the mixture model assumes that categorization difficulty is independent of comparison difficulty. Although there are many cases in which highly related items are quite different in size (e.g., tree-leaf, needle-haystack), one might suspect that pairs with similar magnitudes tend to be somewhat more similar in most respects than do pairs with discrepant magnitudes (e.g., cat-dog). Of course, this confound would invalidate our test of the mixture model if it meant that pairs with a high degree of similarity or association could be categorized more rapidly. This explanation of our results seems quite unlikely for two reasons. First, we obtained the same pattern of results in all four experiments and with two different dimensions. Unless the relation between degree of association and size or height is much stronger than is suggested by our examples, then it seems very unlikely that these two variables would have been confounded in all four experiments. Second, and more importantly, Shoben (1976) demonstrated that similarity had no relation to the latency of the categorization of pairs of exemplars from one category. Tenpenny and Shoben (1992) essentially replicated this finding and observed that their associated pairs took longer to categorize than did nonassociated ones. Thus, there is little reason to believe that the failure of the mixture model occurred because categorization difficulty was confounded with symbolic distance in these experiments.

In contrast to strictly sequential or serial models, parallel models assume that two or more processes overlap. In this paper, we described a race model in which the categorization and computation processes operate in parallel, and a response is determined by the process that is completed first. Distance effects are attenuated for betweencategory pairs because the categorization process determines RT whenever it terminates before the computation process. Although the general pattern of results reported in this paper is consistent with the race model, it does not constitute a positive confirmation of the model, and it is possible that the race model is not correct in every respect. For example, the race model claims that there is not any substantial cost to the use of categorical information because the categorization and comparison processes operate independently. However, in the event that overall capacity is limited, then the duration of the categorization process would be prolonged if the capacity required to complete the comparison process increased with decreasing differences in magnitude (Townsend \& Ashby, 1983). Still, the present results suggest that categorization effects cannot be accounted for without assuming that the operation of categorization and computational processes overlap.

\section{REFERENCES}

BANKs, W. P. (1977). Encoding and processing of symbolic information in comparative judgments. In G. H. Bower (Ed.), The psychology of learning and motivation (Vol. 11, pp. 101-159). New York: Academic Press.

BROWN, N. R., \& SIEGLER, R. S. (1991). Subjective organization of U.S. presidents. American Journal of Psychology, 104, 1-33.

Chang, T. M. (1986). Semantic memory: Facts and models. Psychological Bulletin, 99, 199-220.

CLARK, H. H. (1973). The language-as-a-fixed-effect fallacy: A critique of language statistics in psychological research. Journal of Verbal Learning \& Verbal Behavior, 12, 335-359.

Diaconis, P., \& EFron, B. (1983, May). Computer-intensive methods in statistics. Scientific American, 248, 116-130.

HOLYOAK, K. (1978). Comparative judgments with numerical reference points. Cognitive Psychology, 10, 203-243.

HolyonK, K., \& MAH, W. A. (1982). Cognitive reference points in judgments of symbolic magnitude. Cognitive Psychology, 14, 328352.

HOLYOAK, K., \& PATterson, K. K. (1981). A positional discriminability model of linear order judgments. Journal of Experimental Psychology: Human Perception \& Performance, 7, 1283-1302.

Kosslyn, S. M., Murphy, G. L., Bemesderfer, M. E., \& Feinstein, K. J. (1977). Category and continuum in mental comparisons. Journal of Experimental Psychology: General, 106, 341-375.

KuČERA, F., \& FranCIS, W. (1967). Computational analysis of presentday American English. Providence, RI: Brown University Press.

LiNK, S. W., \& TindalL, A. D. (1971). Speed and accuracy in comparative judgments of line length. Perception \& Psychophysics, 9, 284-288.

LofTUS, E. F., \& SCHEFF, R.W. (1971). Categorization norms for fifty representative instances. Journal of Experimental Psychology, 91, 355-364.

LUCE, R. D. (1986). Response times: Their role in inferring elementary mental organization. New York: Oxford University Press.

MAKı, R. H. (1981). Categorization and distance effects with spatial linear orders. Journal of Experimental Psychology: Learning, Memory, \& Cognition, 7, 15-32.

MOYER, R. S. (1973). Comparing objects in memory: Evidence suggesting an internal psychophysics. Perception \& Psychophysics, 13, 180-184.

NOREEN, E. W. (1989). Computer intensive methods for testing hypotheses: An introduction. New York: Wiley.

Pliske, R. M., \& SMITH, K. H. (1979). Semantic categorization in a linear order problem. Memory \& Cognition, 7, 297-302.

SAILOR, K. M., \& Shoben, E. J. (1993). The effects of real world knowledge on comparative judgment. Journal of Experimental Psychology: Learning, Memory, \& Cognition, 19, 1321-1327.

SHOBEN, E. J. (1976). The verification of semantic relations in a samedifferent paradigm: An asymmetry in semantic memory. Journal of Verbal Learning \& Verbal Behavior, 15, 365-379.

Shoben, E. J., Cech, C. G., Schwanenflugel, P. J., \& Sailor, K. M. (1989). Serial position effects in comparative judgments. Journal of Experimental Psychology: Human Perception \& Performance, 15, 273-286.

Sмітн, E. E. (1978). Theories of semantic memory. In W. K. Estes (Ed.), Handbook of learning and cognitive processes: Vol. 6. Linguistic functions in cognitive theories (pp. 1-56). Hillsdale, $\mathrm{NJ}$ : Erlbaum.

Tenpenny, P. L., \& Shoben, E. J. (1992). Component processes and the utility of the conceptually-driven/data-driven distinction. Journal of Experimental Psychology: Learning, Memory, \& Cognition, 18, 25-42. Thompson, B. (1992, April). The use of statistical significance in re- 
search: Some criticisms and alternatives. Paper presented at the annual meeting of the American Educational Research Association, San Francisco.

TOWNSEND, J. T., \& ASHBy, F. G. (1983). Stochastic modeling of elementary psychological processes. Cambridge: Cambridge University Press.

Wasserman, S., \& Bockenholt, U. (1989). Bootstrapping: Applications to psychophysiology. Psychophysiology, 26, 208-221.

\section{NOTES}

1. In fact, the mixture model is a more general case of the simple sequential model because it assumes that the probability that the categorization process will lead to a response can vary. If this probability is extremely high, then the mixture model will produce results similar to those produced by the simple sequential model.

2. It should be noted that the use of group data could introduce nonlinearities if the value of $p$ was not fairly similar across subjects. To check whether this averaging might introduce different amounts of bias for the three estimates of $p$, we conducted several simulations. The within-category distance effects were multiplied by $1-p$ to give the between-category effects. The value of $p$ was randomly sampled from a specified distribution. The mean of the within-category effects and these between-category effects were calculated, and $p$ was calculated from their ratio. When $p$ was sampled from a normal or uniform distribution, the three estimates of $p$ were quite close and very similar to the mean of the distribution. Even when the distribution was substantially skewed by truncating one of the tails, the three estimates were quite comparable.

3. An idea of how discrepant these results are from results that one would expect to obtain if the two estimates of $p$ were drawn from the same population can be obtained by contrasting them with results from a similar comparison of estimates of $p_{12}$ to other estimates of $p_{12}$ or estimates of $p_{23}$ to other estimates of $p_{23}$. We generated two sets of 1,000 estimates of $p_{12}$ and compared each estimate in the first set with every estimate in the second set. In 100 replications of this resampling plan, the proportion of estimates from the first set that were greater than estimates from the second set ranged from .471 to .521 , with a mean of .498 . An identical analysis of the $p_{23}$ estimate yielded a range of .490-.513, with a mean of .50 .

4. In fact, if each of these experiments and Experiment 2 is treated as an independent event with two possible outcomes (e.g., $p_{12}>p_{23}$, or $p_{12}<p_{23}$ ), the chance probability of obtaining a $p_{12}$ estimate greater than the $p_{23}$ estimate in all four experiments is $p=.0625$.

(Manuscript received July 21, 1994; revision accepted for publication September 1, 1995.) 